Disease Focus

Editor's Note: Disease Focus articles provide brief overviews of a neural disease or syndrome, emphasizing potential links to basic neural mechanisms. They are presented in the hope of helping researchers identify clinical implications of their research. For more information, see http://www.jneurosci.org/misc/ifa_minireviews.dtl.

\title{
Spasmodic Dysphonia: a Laryngeal Control Disorder Specific to Speech
}

\author{
Christy L. Ludlow \\ Department of Communication Sciences and Disorders, James Madison University, Harrisonburg, Virginia 22807
}

Spasmodic dysphonia (SD) is a rare neurological disorder that emerges in middle age, is usually sporadic, and affects intrinsic laryngeal muscle control only during speech. Spasmodic bursts in particular laryngeal muscles disrupt voluntary control during vowel sounds in adductor SD and interfere with voice onset after voiceless consonants in abductor SD. Little is known about its origins; it is classified as a focal dystonia secondary to an unknown neurobiological mechanism that produces a chronic abnormality of laryngeal motor neuron regulation during speech. It develops primarily in females and does not interfere with breathing, crying, laughter, and shouting. Recent postmortem studies have implicated the accumulation of clusters in the parenchyma and perivascular regions with inflammatory changes in the brainstem in one to two cases. A few cases with single mutations in THAP1, a gene involved in transcription regulation, suggest that a weak genetic predisposition may contribute to mechanisms causing a nonprogressive abnormality in laryngeal motor neuron control for speech but not for vocal emotional expression. Research is needed to address the basic cellular and proteomic mechanisms that produce this disorder to provide intervention that could target the pathogenesis of the disorder rather than only providing temporary symptom relief.

\section{Introduction}

Persons affected with spasmodic dysphonia (SD) have involuntary spasms of certain laryngeal muscles that only occur during particular speech items (Shipp et al., 1985; Nash and Ludlow, 1996; Cyrus et al., 2001). In the adductor type, the vocal fold closing (adductor) muscles spasm, closing the vocal folds too tightly and cutting off the voice (Parnes et al., 1978) on words beginning with vowels or on vowels in the middle of words. This type affects at least $80 \%$ of persons with SD and disrupts sentences like, "we eat eggs every day" (Erickson, 2003). The abductor type is

Received May 31, 2010; revised 0ct. 8, 2010; accepted Nov. 1, 2010.

C.L.L. is Chair of the Scientific Advisory Board of the National Spasmodic Dysphonia Association as an unpaid volunteer and receives support as a project leader on National Institutes of Health Grant U54 NS065701, The Dystonia Coalition.

Correspondence should be addressed to Dr. Christy L. Ludlow, James Madison University, Professor of Communication Sciences and Disorder, Director, Laboratory on Neural Bases of Communication and Swallowing, HHS 1141, MSC 4304, Harrisonburg, VA 22807.E-mail: ludlowcx@jmu.edu. DOI:10.1523/JNEUROSCI.2758-10.2011

Copyright $\odot 2011$ the authors $\quad 0270-6474 / 11 / 310793-05 \$ 15.00 / 0$ rarer with uncontrolled spasms in the vocal fold opening (abductor) muscle resulting in breathy bursts when attempting to start voice after voiceless consonants such as /s/, /f/, /h/, /p/, /t/, and /k/ (Rodriquez et al., 1994) and disrupts sentences such as, "he had half a head of hair" (Rontal et al., 1991). Rarely, both types occur in one person. Approximately one-third of persons with $\mathrm{SD}$ also have voice tremor (Schweinfurth et al., 2002), which makes the pitch and loudness of the voice waver at $5 \mathrm{~Hz}$ during vowels and is most evident when " $/ \mathrm{a} /$ " as in the word "all" is produced for at least $5 \mathrm{~s}$ (Schweinfurth et al., 2002).

The disorder develops without warning or any clear antecedent events in middle age. Patients report common occurrences such as upper respiratory infections and stress before onset (Schweinfurth et al., 2002). Usually, the voice disruptions gradually increase over several months then become consistent and remain chronic without further progression (Brin et al.,
1998). Voice production becomes increasingly physically effortful, although no noticeable increase in severity usually occurs. The vast majority of those affected are female, with some estimates as high as $80 \%$ (Adler et al., 1997).

Spasmodic dysphonia is rare; some estimates are as low as 1 per 100,000 cases (Nutt et al., 1988), although accurate diagnosis is a significant roadblock to research. Currently it is not clear whether the disorder has a genetic basis; most cases are sporadic, but case series report as high as $20 \%$ may have other forms of focal dystonia such as writer's cramp (Schweinfurth et al., 2002). Only a very small proportion, generally $<8 \%$, report that other family members are affected with dystonia of any kind (Xiao et al., 2010), and fewer still report having another family member with SD.

\section{Possible disease mechanisms}

One of the mysteries of this disorder it that it is task specific; it only occurs during 
speaking and does not affect emotional expression such as laughter, crying, and shouting (Bloch et al., 1985). This feature was originally thought to suggest that the disorder was psychogenic but is now attributed to the difference between the mammalian vocalization system, which includes isolation cries, alarm calls, sex, and pain, and the human speech system (Fig. 1). Mammalian vocalization can be triggered from the cingulate cortex and the periaquaductal gray to central pattern generators in the pons and brainstem (Jürgens, 2002a,b). Such vocalizations can be environmentally modified but are not learned. In contrast, speech is learned, generative rather than imitative as humans can formulate novel sentences for communication, and integrates with the auditory and voluntary motor control systems (Vihman and de Boysson-Bardies, 1994; MacNeilage, 1998) (Fig. 1b). Only the human has a direct corticobulbar pathway from the laryngeal cortex to the nucleus ambiguus (Kuypers, 1958). Therefore, neural systems involved in learning speech are likely affected in SD (Fig. $1 B$ ), while those involved in emotional vocalization are not (Fig. $1 \mathrm{~A}$ ). To identify the neural abnormalities in SD, differences between these two neural systems (one for emotional vocalization and the other for speech) must account for symptoms being absent in the former and present in the latter.

The left perisylvian neural system for speech and language functions in the cerebral cortex involving the supramarginal gyrus, the arcuate fasciculus, the frontal opercular area, M1, and the internal capsule, has been examined in patients with SD using both structural and functional neuroimaging techniques (Haslinger et al., 2005; Ali et al., 2006; Simonyan et al., 2008; Simonyan and Ludlow, 2010). The laryngeal muscles are bilaterally controlled from both hemispheres (Rödel et al., 2004), making the system vulnerable to unilateral abnormalities interfering with bilateral control of the laryngeal muscles.

Using diffusion tensor imaging, fractional anisotropy was reduced in the genu region of the internal capsule on the right side with bilaterally increased diffusivity in the corticobulbar tract in SD patients compared with controls (Simonyan et al., 2008). Other regions in the basal ganglia also showed group increases in water diffusivity in SD patients on structural imaging. Postmortem tissues from one patient with confirmed SD showed a loss of axonal density and myelin content in genu

\section{A Human Emotional Vocalization System}

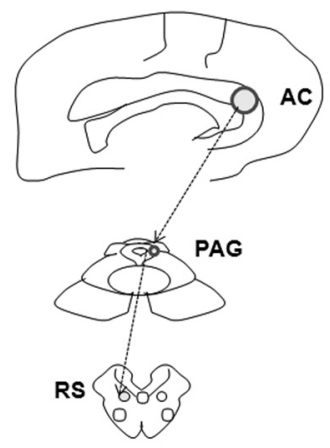

\section{B Human Voice for Speech System}

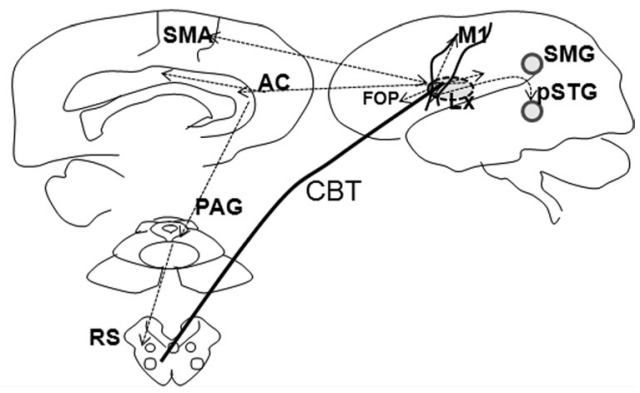

Figure 1. Illustration of the overlap and differences in the neural systems involved in emotional vocalization and in voice production for speech communication. $\boldsymbol{A}$, A schematic diagram of the human emotional vocalization system, which includes the anterior cingulate $(A C)$, the periaquaductal gray (PAG), and the reticular system (RS) in the medulla with input to the laryngeal motor neurons in the nucleus ambiguous, based on the work of Jürgens $(2002 a, b)$ in the squirrel monkey. $\boldsymbol{B}$, A schematic diagram of the human voice for a speech system based on the study by Kuypers (1958), transcranial magnetic stimulation (Rödel et al., 2004), and functional neuroimaging (Schulz et al., 2005; Loucks et al., 2007; Chang et al., 2009; Simonyan et al., 2009), and includes the laryngeal motor cortex (Lx), the direct corticobulbar tract to the motor neurons (CBT), the frontal opercular speech system (FOP), the primary motor cortex (M1), the supplementary motor area (SMA), the posterior superior temporal gyrus (pSTG), and the supramarginal gyrus (SMG).

of the right internal capsule in comparison with postmortem tissues from unaffected controls. Hematoxylin and eosin-stained postmortem sections from the one case showed clusters of dark blue/black basophilic precipitates in the parenchyma and small-caliber vessels in the putamen, globus pallidus, and the posterior limb of the internal capsule in an SD patient, which were absent in three controls. These clusters were positive for calcium and phosphate with single scattered iron deposits. Replication in other postmortem samples from SD patients is needed. It is unclear whether the clusters represent a selflimited process leading to the accumulation of material in the parenchyma or are the result of a disease process that produced an accumulation of material in the parenchyma.

Reticular regions in the brainstem contain central pattern generators for vocal control that are likely activated by both speech and emotional vocalization (Jürgens, 2002a). Postmortem tissues from the brainstem of two SD cases, the one case described above and another with SD and voice tremor, were compared with controls (Simonyan et al., 2010). Small clusters of inflammation (involving microglia) were found in the reticular formation in both patients, but none were found in the controls in regions sur- rounding the solitary tract, spinal trigeminal, nucleus ambiguus, inferior olive, and pyramids. Mild neuronal degeneration and depigmentation were observed in the substantia nigra and locus coeruleus without abnormal protein accumulations, demyelination, or axonal degeneration. Given that brainstem mechanisms serve as the final common pathway for both speech and emotional vocalization, the inflammatory processes in the brainstem are unlikely to be the basis for the symptoms in SD as they would affect both speech and emotional vocalization. Perhaps the brainstem abnormalities serve as an interference with volitional laryngeal control during speech production because of the precise voice onset time requirements for speech sounds that are not required in emotional expression such as laughter and crying. Compensatory abnormalities may have developed in cortical control for speech production systems as the patient attempted to meet precise control demands for speech.

Functional neuroimaging techniques such as positron emission tomography and blood level oxygenation-dependent functional magnetic resonance imaging have been used to examine for differences in function in SD compared with controls (Haslinger et al., 2005; Ali et al., 2006; Simonyan and Ludlow, 2010). As symptom 
production while speaking likely alters brain function in SD, brain differences between SD patients and controls while speaking are difficult to interpret. One approach is to examine brain function differences from controls when patients are asymptomatic to determine whether there are differences in brain function independent of symptom expression. This was done in SD after treatment with botulinum toxin injections into the laryngeal muscle to reduce the involuntary muscle spasms (Haslinger et al., 2005; Ali et al., 2006); however, voice production is not completely normal with treatment (Paniello et al., 2008). The effects of botulinum toxin on the laryngeal muscle contractions cannot be assumed, however, to have altered only muscle spasms. There may be retrograde transport affecting input to the laryngeal motor neurons (Moreno-López et al., 1997; Antonucci et al., 2008). In addition, as the muscle spasms are reduced not only in the laryngeal muscle injected but also in other laryngeal muscles on the opposite side of the larynx (Bielamowicz and Ludlow, 2000), the sensory feedback from the larynx is altered by less mucosal compression and lower subglottal pressures in the trachea due to reduced hyperadduction during speech.

To identify which parts of the speech system are affected in SD, nonspeech tasks such as whimpering and coughing were compared between SD and controls ( $\mathrm{Si}$ monyan and Ludlow, 2010). Similar functional differences from controls occurred during whimpering and speech; activity in the somatosensory region was increased in the patients compared with controls and related to symptom severity. It is unclear whether brain function differences from controls are the result of the disorder rather than precursors to it.

\section{Treatments for spasmodic dysphonia}

Symptom reduction occurs when the kinetic output of the laryngeal muscles is reduced either by unilateral recurrent laryngeal nerve section (Dedo, 1976), or by botulinum injections into the adductor muscles for adductor SD or the abductor muscle for abductor SD (Blitzer et al., 1998). Following nerve section, benefits occur until reinnervation takes place and symptoms return (Dedo, 1976; Aronson and DeSanto, 1981; Fritzell et al., 1982). Further approaches were to avulse a long section of the recurrent nerve to prevent reinnervation (Netterville et al., 1991; Weed et al., 1996) or bilaterally section and reinnervate the nerve branch going to the thyroarytenoid muscle to the ansa cervicalis to prevent reinnervation by the recurrent laryngeal nerve (Berke et al., 1999; Chhetri et al., 2006). In these treatments, a balance must be achieved between adequately reducing vocal fold hyperadduction while not producing aspiration during swallowing or aphonic speech (Salassa et al., 1982).

These treatment approaches interfere with muscle action rather than blocking abnormal interneuron firing patterns in the laryngeal efferent pathway. As mentioned earlier, symptom reduction might be due to alterations in sensory feedback to the CNS (Bielamowicz and Ludlow, 2000) or retrograde transmission of botulinum toxin to modulate interneurons in the CNS affecting motor neuron firing (Moreno-López et al., 1997; Antonucci et al., 2008). Clinically, after unilateral injections of botulinum toxin into the thyroarytenoid muscle in adductor SD, the numbers of spasms in the untreated muscles on the opposite side of the larynx were reduced (Bielamowicz and Ludlow, 2000). Reduced muscle force in the larynx may reduce sensory feedback to the central pattern generators in the brainstem or the sensorimotor cortex. One study of SD patients before and after successful treatment with botulinum toxin injection found increased activity in the sensorimotor cortex in untreated SD patients, which normalized after treatment with botulinum toxin muscle injection (Ali et al., 2006).

\section{Disease mechanisms that need to be explored in SD}

Functional neuroimaging studies are difficult to interpret in SD. However, some characteristics of the disorder suggest some potential neural bases for the disorder. First, onset is gradual after which the disorder becomes chronic, suggesting some adaptation processes are involved in the development of the pathophysiology. Although only the speech production system is involved, speech is normal during whispering in SD and symptoms are dramatically reduced after botulinum toxin injection, suggesting that the speech production system is not permanently altered as it can be normalized immediately with changes in laryngeal output. One interpretation could be that the increased cortical activation in the motor and sensory laryngeal cortices found on functional neuroimaging (Ali et al., 2006; Simonyan and Ludlow, 2010) may have developed in response to pathophysiology elsewhere in the neural laryngeal control system and might represent compensatory mechanisms responding to interference with laryngeal muscle control downstream. The neuropathology findings in the single postmortem case (Simonyan et al., 2008) may indicate abnormalities affecting either the internal capsule affecting the corticobulbar pathway, the basal ganglia, or feedback loops that modulate cortical control. Perturbation studies in normal speakers have demonstrated that speech gestures respond rapidly to online changes in both articulator position (Abbs and Gracco, 1984) and auditory feedback (Larson et al., 2000) within $<100$ ms when there is a mismatch between the expected and altered feedback. Further, the lack of symptoms during whispering when the vocal folds are not vibrating suggests that changes in laryngeal sensory feedback either from the vocal fold mucosa or subglottal pressures in the trachea may play a role in the pathophysiology of the disorder. One hypothesis, then, might be that the pathophysiology may involve sensory feedback from the laryngeal periphery affecting cortical physiology in SD. Further research on the role of laryngeal sensory feedback in the manipulation of symptoms needs to be performed.

The recent discovery of THAP (thanatos-associated protein) domain-containing apoptosis-associated protein 1 (THAP1) as being the basis for DYT6 dystonia (Bressman et al., 2009; Fuchs et al., 2009) has led to studies of THAP1 mutations in primary focal dystonias of early onset (Houlden et al., 2010) and late onset (Xiao et al., 2010). Some studies have identified THAP1 mutations associated with generalized dystonia, which may first affect the larynx (Djarmati et al., 2009), while one study has reported on SD adult onset that did not progress to a generalized form of dystonia (Xiao et al., 2010). Possibly, a variety of mutations in THAP1 may play a role in the development of cervicocranial dystonias including SD (Ozelius and Bressman, 2010). However, the role of THAP1 mutations in SD is somewhat limited; of 460 patients with SD screened for sequence variants in three exons of THAP1 only $5(1 \%)$ had mutations in THAP1. The SD patients with THAP1 mutations who had adductor SD were female with a mean onset age of 57.8 years. Two had single amino acid substitutions (p.F132S and p.A166T). As SD is often confused with other voice disorders (Ludlow et al., 2008; Roy et al., 2008), further study in well documented SD patients with clear phenotype characterization is warranted. 
The putative transcriptional dysregulation produced by THAP1 needs to be determined for each of the coding mutations identified thus far in the THAP1 gene, with nine coding mutations documented in one study on late-onset cases (Xiao et al., 2010), and nine others in another study on earlyonset dystonia (Houlden et al., 2010). The functional consequences of these mutations in the mature nervous system need to be determined, and the possible mechanisms of neuronal disruption that could lead to abnormalities in the control of motor-neuron firing need to be determined (Tamiya, 2009).

The histopathology identified in the one postmortem case of spasmodic dysphonia raises questions about transcription dysregulation that could result in the accumulation of parenchymal clusters located close to the vessel walls (Simonyan et al., 2008). Such material, if found in other cases, needs to be studied to determine the proteomic composition.

THAP1 models are limited to cell cycle pathways in humans, fish, and nematodes, and appear to be critical regulators of cell proliferation and cell cycle progression (Bessière et al., 2008). THAP1 mutations need to be developed in mammalian models to examine the effects of these mutations on the mammalian laryngeal system. Attention should be given to selflimiting mechanisms for focal adult onset dystonias including SD. The mechanisms involved must differ from the neurodegenerative disorders such as Parkinson disease and amyotrophic lateral sclerosis. Because no clear pathological inclusions such as Lewy bodies have yet been identified in focal dystonia, pathologic confirmation of the disease is not available and thus far only symptomatology is available for diagnosis. Research on possible basic mechanisms is clearly needed, and additional postmortem tissue amenable to proteomic analysis is a necessary first step. The possible role of THAP1 mutations needs to be explored.

In conclusion, the level of knowledge of the pathologic mechanisms and the pathways involved in this and other focal dystonias is limited compared with progressive neurodegenerative disorders. As the disorder is not progressive yet results in a chronic disability, a different type of molecular mechanism is likely involved and needs to be determined.

\section{References}

Abbs JH, Gracco VL (1984) Control of complex motor gestures: orofacial muscle responses to load perturbations of the lip during speech. J Neurophysiol 51:705-723.
Adler CH, Edwards BW, Bansberg SF (1997) Female predominance in spasmodic dysphonia. J Neurol Neurosurg Psychiatry 63:688.

Ali SO, Thomassen M, Schulz GM, Hosey LA, Varga M, Ludlow CL, Braun AR (2006) Alterations in CNS activity induced by botulinum toxin treatment in spasmodic dysphonia: an H215O PET study. J Speech Lang Hear Res 49:1127-1146.

Antonucci F, Rossi C, Gianfranceschi L, Rossetto O, Caleo M (2008) Long-distance retrograde effects of botulinum neurotoxin A. J Neurosci 28:3689-3696.

Aronson AE, DeSanto LW (1981) Adductor spastic dysphonia: $11 / 2$ years after recurrent laryngeal nerve resection. Ann Otol Rhinol Laryngol 90:2-6.

Berke GS, Blackwell KE, Gerratt BR, Verneil A, Jackson KS, Sercarz JA (1999) Selective laryngeal adductor denervation-reinnervation: a new surgical treatment for adductor spasmodic dysphonia. Ann Otol Rhinol Laryngol 108:227-231.

Bessière D, Lacroix C, Campagne S, Ecochard V, Guillet V, Mourey L, Lopez F, Czaplicki J, Demange P, Milon A, Girard JP, Gervais V (2008) Structure-function analysis of the THAP zinc finger of THAP1, a large $\mathrm{C} 2 \mathrm{CH}$ DNA-binding module linked to Rb/E2F pathways. J Biol Chem 283:4352-4363.

Bielamowicz S, Ludlow CL (2000) Effects of botulinum toxin on pathophysiology in spasmodic dysphonia. Ann Otol Rhinol Laryngol 109:194-203.

Blitzer A, Brin MF, Stewart CF (1998) Botulinum toxin management of spasmodic dysphonia (laryngeal dystonia): a 12-year experience in more than 900 patients. Laryngoscope 108:14351441.

Bloch CS, Hirano M, Gould WJ (1985) Symptom improvement of spastic dysphonia in response to phonatory tasks. Ann Otol Rhinol Laryngol 94:51-54.

Bressman SB, Raymond D, Fuchs T, Heiman GA, Ozelius LJ, Saunders-Pullman R (2009) Mutations in THAP1 (DYT6) in early-onset dystonia: a genetic screening study. Lancet Neurol 8:441-446.

Brin MF, Blitzer A, Stewart C (1998) Laryngeal dystonia (spasmodic dysphonia): observations of 901 patients and treatment with botulinum toxin. Adv Neurol 78:237-252.

Chang SE, Kenney MK, Loucks TM, Poletto CJ, Ludlow CL (2009) Common neural substrates support speech and non-speech vocal tract gestures. Neuroimage 47:314-325.

Chhetri DK, Mendelsohn AH, Blumin JH, Berke GS (2006) Long-term follow-up results of selective laryngeal adductor denervationreinnervation surgery for adductor spasmodic dysphonia. Laryngoscope 116:635-642.

Cyrus CB, Bielamowicz S, Evans FJ, Ludlow CL (2001) Adductor muscle activity abnormalities in abductor spasmodic dysphonia. Otolaryngol Head Neck Surg 124:23-30.

Dedo HH (1976) Recurrent laryngeal nerve section for spastic dysphonia. Ann Otol Rhinol Laryngol 85:451-459.

Djarmati A, Schneider SA, Lohmann K, Winkler S, Pawlack H, Hagenah J, Brüggemann N, Zittel S, Fuchs T, Raković A, Schmidt A, Jabusch HC, Wilcox R, Kostić VS, Siebner H, Alten- müller E, Münchau A, Ozelius LJ, Klein C (2009) Mutations in THAP1 (DYT6) and generalised dystonia with prominent spasmodic dysphonia: a genetic screening study. Lancet Neurol 8:447-452.

Erickson ML (2003) Effects of voicing and syntactic complexity on sign expression in adductor spasmodic dysphonia. Am J Speech Lang Pathol 12:416-424.

Fritzell B, Feuer E, Haglund S, Knutsson E, Schiratzki H (1982) Experiences with recurrent laryngeal nerve section for spastic dysphonia. Folia Phoniat 34:160-167.

Fuchs T, Gavarini S, Saunders-Pullman R, Raymond D, Ehrlich ME, Bressman SB, Ozelius LJ (2009) Mutations in the THAP1 gene are responsible for DYT6 primary torsion dystonia. Nat Genet 41:286-288.

Haslinger B, Erhard P, Dresel C, Castrop F, Roettinger M, Ceballos-Baumann AO (2005) "Silent event-related" fMRI reveals reduced sensorimotor activation in laryngeal dystonia. Neurology 65:1562-1569.

Houlden H, Schneider SA, Paudel R, Melchers A, Schwingenschuh P, Edwards M, Hardy J, Bhatia KP (2010) THAP1 mutations (DYT6) are an additional cause of early-onset dystonia. Neurology 74:846-850.

Jürgens U (2002a) Neural pathways underlying vocal control. Neurosci Biobehav Rev 26:235-258.

Jürgens U (2002b) A study of the central control of vocalization using the squirrel monkey. Med Eng Phys 24:473-477.

Kuypers HG (1958) Cortico-bulbar connexions to the pons and lower brainstem in man. An anatomical study. Brain 81:364-388.

Larson CR, Burnett TA, Kiran S, Hain TC (2000) Effects of pitch-shift velocity on voice Fo responses. J Acoust Soc Am 107:559-564.

Loucks TM, Poletto CJ, Simonyan K, Reynolds CL, Ludlow CL (2007) Human brain activation during phonation and exhalation: common volitional control for two upper airway functions. Neuroimage 36:131-143.

Ludlow CL, Adler CH, Berke GS, Bielamowicz SA, Blitzer A, Bressman SB, Hallett M, Jinnah HA, Juergens U, Martin SB, Perlmutter JS, Sapienza C, Singleton A, Tanner CM, Woodson GE (2008) Research priorities in spasmodic dysphonia. Otolaryngol Head Neck Surg 139:495-505.

MacNeilage PF (1998) The frame/content theory of evolution of speech production. Behav Brain Sci 21:499-511.

Moreno-López B, Pastor AM, de la Cruz RR, Delgado-García JM (1997) Dose-dependent, central effects of botulinum neurotoxin type A: a pilot study in the alert behaving cat. Neurology 48:456-464.

Nash EA, Ludlow CL (1996) Laryngeal muscle activity during speech breaks in adductor spasmodic dysphonia. Laryngoscope 106:484489.

Netterville JL, Stone RE, Rainey C, Zealear DL, Ossoff RH (1991) Recurrent laryngeal nerve avulsion for treatment of spastic dysphonia. Ann Otol Rhinol Laryngol 100:10-14.

Nutt JG, Muenter MD, Aronson A, Kurland LT, Melton LJ 3rd (1988) Epidemiology of focal and generalized dystonia in Rochester, Minnesota. Mov Disord 3:188-194. 
Ozelius LJ, Bressman SB (2010) THAP1: role in focal dystonia? Neurology 74:192-193.

Paniello RC, Barlow J, Serna JS (2008) Longitudinal follow-up of adductor spasmodic dysphonia patients after botulinum toxin injection: quality of life results. Laryngoscope 118:564-568.

Parnes SM, Lavorato AS, Myers EN (1978) Study of spastic dysphonia using videofiberoptic laryngoscopy. Ann Otol Rhinol Laryngol 87:322-326.

Rödel RM, Olthoff A, Tergau F, Simonyan K, Kraemer D, Markus H, Kruse E (2004) Human cortical motor representation of the larynx as assessed by transcranial magnetic stimulation (TMS). Laryngoscope 114:918922.

Rodriquez AA, Ford CN, Bless DM, Harmon RL (1994) Electromyographic assessment of spasmodic dysphonia patients prior to botulinum toxin injection. Electromyogr Clin Neurophysiol 34:403-407.

Rontal M, Rontal E, Rolnick M, Merson R, Silverman B, Truong DD (1991) A method for the treatment of abductor spasmodic dysphonia with botulinum toxin injections: a preliminary report. Laryngoscope 101:911-914.

Roy N, Whitchurch M, Merrill RM, Houtz D, Smith ME (2008) Differential diagnosis of adductor spasmodic dysphonia and muscle tension dysphonia using phonatory break analysis. Laryngoscope 118:2245-2253.

Salassa JR, DeSanto LW, Aronson AE (1982) Respiratory distress after recurrent laryngeal nerve section for spastic dysphonia. Laryngoscope 92:240-245.

Schulz GM, Varga M, Jeffires K, Ludlow CL, Braun AR (2005) Functional neuroanatomy of human vocalization: an H215O PET study. Cereb Cortex 15:1835-1847.

Schweinfurth JM, Billante M, Courey MS (2002) Risk factors and demographics in patients with spasmodic dysphonia. Laryngoscope 112:220-223

Shipp T, Izdebski K, Reed C, Morrissey P (1985) Intrinsic laryngeal muscle activity in a spastic dysphonic patient. J Speech Hear Disord 50:54-59.

Simonyan K, Ludlow CL (2010) Abnormal activation of the primary somatosensory cortex in spasmodic dysphonia: an fMRI study. Cereb Cortex 20:2749-2759.

Simonyan K, Tovar-Moll F, Ostuni J, Hallett M, Kalasinsky VF, Lewin-Smith MR, Rushing EJ, Vortmeyer AO, Ludlow CL (2008) Focal white matter changes in spasmodic dysphonia: a combined diffusion tensor imaging and neuropathological study. Brain 131:447-459.
Simonyan K, Ostuni J, Ludlow CL, Horwitz B (2009) Functional but not structural networks of the human laryngeal motor cortex show left hemispheric lateralization during syllable but not breathing production. J Neurosci 29:14912-14923.

Simonyan K, Ludlow CL, Vortmeyer AO (2010) Brainstem pathology in spasmodic dysphonia. Laryngoscope 120:121-124.

Tamiya G (2009) Transcriptional dysregulation: a cause of dystonia? Lancet Neurol 8:416418.

Vihman MM, de Boysson-Bardies B (1994) The nature and origins of ambient language influence on infant vocal production and early words. Phonetica 51:159-169.

Weed DT, Jewett BS, Rainey C, Zealear DL, Stone RE, Ossoff RH, Netterville JL (1996) Longterm follow-up of recurrent laryngeal nerve avulsion for the treatment of spastic dysphonia. Ann Otol Rhinol Laryngol 105:592-601.

Xiao J, Zhao Y, Bastian RW, Perlmutter JS, Racette BA, Tabbal SD, Karimi M, Paniello RC, Wszolek ZK, Uitti RJ, Van Gerpen JA, Simon DK, Tarsy D, Hedera P, Truong DD, Frei KP, Dev Batish S, Blitzer A, Pfeiffer RF, Gong S, LeDoux MS (2010) Novel THAP1 sequence variants in primary dystonia. Neurology 74: $229-238$. 